\begin{tabular}{|c|l|}
\hline Title & $\begin{array}{l}\text { Male specific flight apparatus development in A cyrthosi phon pisum (A phididae, Hemi ptera, Insecta): comparison with } \\
\text { female wing polyphenism }\end{array}$ \\
\hline Author(s) & Ogawa, Kota; Ishikawa, A sano; Kanbe, Takashi; A kimoto, Shin-ichi; Miura, Toru \\
\hline Citation & $\begin{array}{l}\text { Zoomorphology, 131(3), 197-207 } \\
\text { https:/doi.org/10.1007/300435-012-0154-3 }\end{array}$ \\
\hline Issue Date & 2012-09 \\
\hline Doc URL & http://hdl.handle.net/2115/53169 \\
\hline Rights & The original publication is available at www.springerlink.com \\
\hline Type & article (author version) \\
\hline File Information & Zoo131-3_197-207.pdf \\
\hline
\end{tabular}

Instructions for use 


\title{
Male-specific flight-apparatus development in Acyrthosiphon pisum (Aphididae, Hemiptera, Insecta): Comparison with female wing polyphenism
}

\author{
Kota Ogawa ${ }^{1}$, Asano Ishikawa ${ }^{1}$, Takashi Kanbe ${ }^{2}$, Shin-ichi Akimoto ${ }^{2}$ and Toru \\ Miura $^{1 *}$ \\ ${ }^{1}$ Laboratory of Ecological Genetics, Graduate School of Environmental Science, \\ Hokkaido University, Sapporo, Hokkaido 060-0810, Japan \\ ${ }^{2}$ Systematic Entomology, Department of Ecology and Systematics, Graduate \\ School of Agriculture, Hokkaido University, Sapporo, Hokkaido 060-8589, Japan \\ *Author for correspondence (email: miu@ees.hokudai.ac.jp)
}

\begin{abstract}
Wing polymorphisms observed in many Insecta are important topics in developmental biology and ecology; these polymorphisms are a consequence of tradeoffs between flight and other abilities. The pea aphid, Acyrthosiphon pisum, possesses 2 types of wing polymorphisms: one is a genetic wing polymorphism occurring in males and the other is an environmental wing polyphenism seen in viviparous females. Although genetic and environmental cues for the 2 wing polymorphisms have been studied, differences in their developmental regulation have not been elucidated. In particular, there is little knowledge regarding the developmental processes in male wing polymorphism. Therefore, in this study, the development of flight apparatuses and external morphologies were compared among 3 male wing morphs (winged, wingless, and intermediate). These male developmental processes were subsequently compared with those of female wing morphs. Developmental differences between the male and female polymorphisms were identified in flight muscle development and degeneration but not in wing bud development. Furthermore, the nymphal periods of wingless and intermediate males were significantly shorter than that of winged males, indicating the adaptive significance of male winglessness. Overall, this study indicates that the male and female wing polymorphisms are based on different regulatory systems for flight apparatus development, which are probably the result of different adaptations under different selection pressures.
\end{abstract}


Keywords: Wing polymorphism, Flight muscle, Wing bud, Trade-off,

Postembryonic development, Acyrthosiphon pisum

\section{INTRODUCTION}

The enormous diversity of insects, which is derived from their evolutionary success, can be attributed to their acquisition of flight, which is thought to have occurred only once in the insect lineage (Roff 1990; Dudley 2002). Although flight greatly improves the ability of insect species to search for new habitats, mates, and food, flight also incurs large costs for the insect (Harrison 1980; Roff 1990). Consequently, many insect species have secondarily lost flight ability to allocate their saved energy towards other traits (Harrison 1980; Roff 1990; Roff and Fairbairn 1991). Some species exhibit wing polymorphisms in which winged and wingless phenotypes are produced based on genetic or environmental factors (Wagner and Liebherr 1992; Zera and Denno 1997). In some aphid lineages, both genetic wing polymorphisms and environmental polyphenisms are seen even within a single species (Braendle et al. 2006; Brisson 2010).

Although most aphid species exhibit various phenotypes associated with their life cycles, wing polymorphism/polyphenism is one of the most representative phenotypic changes. In both genetic wing polymorphisms and environmentally-induced wing polyphenisms, the winged morph has a welldeveloped thorax with fore- and hindwings whereas the wingless morph has a thinner thorax and lacks wings. Because the ancestral aphid species is thought to have winged adults, the wingless adults are thought to have evolved secondarily (Miyazaki 1987; Dixon 1998; Braendle et al. 2006). The pea aphid, Acyrthosiphon pisum (Harris, 1776), is a species that possesses both wing polyphenism and a genetic wing polymorphism (Brisson 2010). Therefore, this species is suitable for comparing the 2 genetic and environmental wing polymorphisms.

Similar to other aphid species, A. pisum shows thelytokous and viviparous reproduction from spring to fall; in late fall, in response to short-day lengths, they produce males and oviparous females that perform sexual reproduction (Marcovitch 1923; Lamb and Pointing 1972; Dixon 1998; Fig. 1a). In the summer generations of viviparous females, environmental conditions such as high population density or the existence of predators induces the winged phenotype, 
which is thus regarded as a wing polyphenism (Lees 1966; Dixon 1998). On the other hand, different male phenotypes (with or without wings) are determined by a genetic system (Smith and MacKay 1989; Caillaud et al. 2002; Braendle et al. 2005a, b).

A. pisum have an $\mathrm{XO}$ sex-determination system, and the male wing polymorphism is determined by a single gene locus on the X-chromosome called aphicarus (api) (Orlando 1974; Smith and MacKay 1989; Caillaud et al. 2002; Braendle et al. 2005a). Therefore, depending on the maternal genotype, male progeny can be all winged, $50 \%$ winged and $50 \%$ wingless, or all wingless (Smith and MacKay 1989; Braendle et al. 2005a, b).

Using a microarray, Brisson et al. (2007) showed that both the female wing polyphenism and the male wing polymorphism share a series of transcripts, suggesting that males may have co-opted the genetic and/or developmental programs used by the female wing polyphenism. However, no studies comparing the detailed developmental or histological processes of wing formation between the 2 types of wing polymorphisms have been conducted. Furthermore, although comparisons between the external morphologies of winged and wingless morphs have been made with respect to the wing polyphenism, only one previous study comparing wing types in the genetic male polymorphism has been conducted so far (Kring 1977). Therefore, this study focuses on comparing the developmental processes and external morphologies among wing morphs in the male wing polymorphism. In addition, the development of flight apparatuses was compared with that of the wing polyphenism seen in parthenogenetic females. We used 2 informative A. pisum strains: one of which produces both winged and wingless males and the other of which produces both winged and winged-wingless intermediate males, probably according to $\mathrm{X}$-chromosome assignment (see Results for details). Furthermore, previous studies hypothesize that there are some trade-offs of wing development in the male polymorphism, although no clear evidence of this exists (Sack and Stern 2007). In this study, we compared developmental schedule among 3 male wing morphs, to examine whether tradeoffs between dispersal ability and life-history trait and discussed the evolution of wing polymorphism in aphid males. 


\section{MATERIALS AND METHODS}

\section{Insects}

The 06D and HKD strains, whose host plants are alfalfa (Medicago sativa) and red clover (Trifolium pratense), respectively, were collected in Sapporo, Japan and used as focal strains in this study. Stock aphid populations were maintained for several generations under long-day conditions $\left(16 \mathrm{~L} / 8 \mathrm{D}, 20^{\circ} \mathrm{C}\right)$ in test tubes (diameter: $2.5 \mathrm{~cm}$, height: $10 \mathrm{~cm}$ ) containing vetch seedlings (Vicia faba) grown on wet vermiculite (Wilkinson and Ishikawa 2000).

Regarding the male wing types, the 06D strain produces winged (WD) and wingless (WL) males in a 1:1 ratio, while the HKD strain produces winged (WD) and malformed (IM) males in a 1:1 ratio (Fig. 1b). The malformed males possess deformed wings exhibiting intermediate morphology between the winged and wingless males and are thus termed "intermediates" (IM).

\section{Male induction and wing-type determination}

We only used wingless viviparous females for male induction because our preliminary observations found that in the 2 focal strains, winged viviparous females did not produce males even under short-day conditions. Therefore, wingless adult females were prepared by isolation (i.e., low-density conditions) on a vetch seedling across several generations (Ishikawa et al. 2008).

Since the switch to sexual reproduction in Acyrthosiphon pisum (Harris, 1776) (Aphididae, Hemiptera, Insecta) is triggered by short-day lengths and low temperatures (Lamb and Pointing 1972), first-instar aphids produced by a single wingless mother were individually reared on seedlings at $15^{\circ} \mathrm{C}$ under $8 \mathrm{~L} / 16 \mathrm{D}$ conditions. Under these conditions the schedules of morph production can differ between strains (Fig. S1), although males and viviparous and oviparous females are produced across several generations (Ishikawa et al. 2012). In the 06D strain, parental wingless viviparous females produced viviparous progeny approximately during the first 12 days after the first larviposition (Fig. S1a). Males were then exclusively produced until the end of the larviposition period (Fig. S1a). In the HKD strain, males were exclusively produced after the first 16 days from the first larviposition when viviparous females were produced (Fig. S1b). In both strains, 
oviparous females were produced by viviparous females in subsequent generations when they were continuously reared under the same conditions (data not shown). We only used presumptively male aphids according to these schedules for this study. The wing-type of male nymphs was discernible by their strain and wing-bud development (see Results).

\section{Morphometric analysis}

In the case of wing polyphenism in viviparous females, wing morphs are known to exhibit various morphological characteristics (Miyazaki 1987; Braendle et al. 2006; Ishikawa and Miura 2007); however, except Kring (1977), no studies have been conducted to determine whether this is also the case in the male polymorphism. Therefore, in order to elucidate the morphological differences among winged, wingless, and intermediate males, the following body parts of each morph were measured using an image-analyzing system (HIM-1N; HOGA, Osaka, Japan): head width (including compound eye height), mesonotum width and length, and right hind tibial length. In addition, other morphological characteristics (e.g., ocelli and secondary rhinaria) and the coloration of thoracic dorsum (notum) were also examined.

\section{Scanning electron microscopy}

Observations of the thoracic structures where wing buds develop in WD or IM types were carried out by scanning electron microscopy. Aphid samples of all instars were fixed in FAA solution (formalin:ethanol:acetic acid, 6:16:1) and transferred into increasing concentrations of ethanol followed by $t$-butanol. After that, they were freeze-dried using an ES-2030 freeze dryer (Hitachi, Tokyo) and coated with gold ions with an E-1010 ion sputter (Hitachi, Tokyo). The detailed morphological characteristics of thoracic structures were observed with a scanning electron microscope (JSM-5510LV; JEOL, Tokyo). 


\section{Histological examination}

To compare the developmental processes of the thoracic region of each morph (WD, WL, and IM), paraffin sections of third- to fifth-instar aphids were made as described previously (Ishikawa et al. 2008). Specimens were fixed in FAA fixative, dehydrated in increasing concentrations of ethanol, and transferred into xylene before being embedded in paraffin. Serial sections $(5-\mu \mathrm{m}$ thick) were processed routinely and stained with hematoxylin and eosin. Tissues were observed under a light microscope (BX-51; Olympus, Tokyo), and images were captured using a CCD camera (DP-72; Olympus) and DP2-BSW software (Olympus). 3D structures of flight muscles were reconstructed using a free software package (Delta Viewer, version 2.1.1, http://delta.math.sci.osakau.ac.jp/DeltaViewer/).

\section{Comparison of nymphal periods}

We measured the nymphal periods of males to examine whether they are shortened in non-winged phenotypes (WL and IM). Since all aphid species (Aphididae) possess 5 postembryonic instars including the adult stage (Blackman 1987; Dixon 1998), nymphal instars were checked daily to measure stadia, including the first-second- (the first and second stadia were combined), third-, and fourth-stadia, in addition to the overall nymphal period. To avoid the negative effects of nutritional conditions, vetch seedlings were renewed every few days. The wing-types were discriminated after the imaginal molt.

\section{Observations of testes}

To examine whether spermatogenesis was properly carried out in IM males, testes were observed. Testes from third- and fourth-instar nymphs, and adults were isolated in phosphate-buffered saline (PBS), fixed in $4 \%$ paraformaldehyde in PBS for $30 \mathrm{~min}$, and finally washed again in PBS. To clarify the testis structure, F-actin was stained with 5 units/mL Oregon Green 514 phalloidin (Molecular Probes, Eugene) for $60 \mathrm{~min}$. The samples were then washed and mounted in Vectashield (Vector Laboratories, Burlingame). Observations were carried out 
using a Carl Zeiss LSM510 META laser-scanning confocal microscope equipped with a laser unit: Argon/2 and DPSS 561-10, HeNe633.

\section{RESULTS}

\section{Morphometric analysis}

To examine morphological differences among the 3 morphs in males (WD, WL, and IM), we compared head and mesonotum width, mesonotum and hind tibial length, notum color (i.e., the thoracic segment), and the sensory organs of fifthinstar aphids. Head width and hind tibial length did not significantly differ among morphs (Table 1, Tukey-Kramer test, $p>0.05$ ). The mesonotum width of WL males was significantly smaller than those of the others, and the mesonotum width of IM males was in between those of the WD and WL morphs (Table 1, TukeyKramer test, $p<0.05)$. The mesonotum length was also significantly different between groups; WL males had the shortest mesonotum lengths, and WD males had the longest ones (Table 1, Tukey-Kramer test, $p<0.05$ ). The thoracic segments of WD males were pigmented a dark-brown color (Fig. 2a), whereas the thoraces of WL males were more greenish like other body parts (Fig. 2e). The thoracic pigmentation of IM males was intermediate level between WD and WL (Fig. 2b-d). All male morphs possessed fully developed ocelli and well-developed sensory organs called "secondary rhinaria" on their antennae (Fig. S2; Kring 1977).

\section{Wing bud development}

There were no obvious variations in the external morphologies of the first- and second-instar male nymphs of both strains (Fig. 3a, b). In the third instar, small wing buds were observed in about half of the males of the 06D strain (Fig. 3c), while the other half did not exhibit any swollen structures (Fig. 3e). Thus, based on these structures, WD and WL males were discernible at the third instar. On the other hand, more than half of the third-instar males of the HKD strain possessed wing buds (Fig. 3d). Since the half of the HKD strain males were WD and the 
other were IM, IM males were thought to show variations in their wing-bud development.

In fourth-instar WD males of both strains, wing buds were enlarged (called "wing pads") (Fig. 3f; Ishikawa et al. 2008), while deformed and misshaped wing buds/pads were observed in IM males (Fig. 3g, h). No wing buds/puds were seen in WL males (Fig. 3i).

Fifth-instar WD males (i.e. adults), had completely formed wings and well-developed thoraces (Fig. 3j), whereas WL males had thin thoraces without wings (Fig. 3m). The IM adult males possessed defective wings or swollen structures similar to wing buds (Fig. 3k, l) and exhibited some variations in wing number (1-4). Interestingly, hindwings were well developed relative to forewings (Fig. 2b-d; 3g, h, k, 1).

\section{Inner thoracic structures}

In histological examinations, we used third-, fourth-, and fifth-instar males because their wing types could be distinguished by external morphologies. Unfortunately, based on the external morphologies, we were not able to identify wing types of first- and second-instar males.

In the male wing polymorphism, the following types of flight muscles were observed in all morphs (Fig. 4): dorsoventral muscle (DVM), dorsal longitudinal muscle (DLM), and oblique dorsal muscle (ODM) (Fig. 4f, g). The wing primordia (epithelia) and flight muscles were recognized at the third instar in WD and IM males (Fig. 4a, b). At this stage, the wing primordia were almost degenerated in WL males (Fig. 4c) and flight muscles were less developed than the other 2 morphs (Fig. 4c). At the fourth instar, the flight muscles of WD males were largely developed and occupied more than half of the thoracic volume (Fig. 4a). The flight muscles of IM and WL males were not as well developed as those of WD males (Fig. 4b, c). The wing buds (or pads) of WD and IM males exhibited sheath-like shapes in which the wing epithelia were folded in complicated structures (Fig. 4a, b); however, these were not seen in WL males (Fig. 4c). The transverse sections of the overall thoraces of fifth-instar WD males exhibited long ellipsoid shapes (i.e., the dorsoventral height increased) in which well-developed flight muscles were observed (Fig. 4a); those in WL and IM males were more 
circular (Fig. 4b, c). IM males exhibited developed flight muscles that occupied more than half of the thoracic segments, although the extent of development appeared to be less than that of WD males (Fig. 4b). The DVMs of IM males were crooked and were awkwardly shaped probably because their flight muscle development was restricted by the overall thoracic size. Flight muscles were also seen in WL males (Fig. 4c), although the degree of development differed among individuals (Fig. S4). In general, the flight muscles of adult WL males tend to resemble those of third-instar WD males. Moreover, the flight muscles of adult IM males appear similar to those of fourth-instar WD males.

Greater details of muscle cell differentiation can be seen histologically on cross sections. This process was observed while focusing on the DVMs (Fig. 5). In the case of WD males, precursor muscle cells, i.e., myoblasts, which had relatively large nuclei and were densely stained with hematoxylin, were seen at the third instar (Fig. 5a left); they proliferated until the fourth instar. During the fourth instar, proliferation appeared to be complete, indicating that numerous muscle fibers were formed (Fig. 5a middle). At this stage, muscle cells fuse together to form syncytial cells. After the final molt to the fifth instar, the muscles were well developed and numerous muscle fibers were clearly observed (Fig. 5a right). Nearly identical processes of muscle-cell differentiation were also observed in WL (Fig. 5c) and IM (Fig. 5b) morphs.

\section{Nymphal period}

In order to examine whether wing type affects postembryonic growth, nymphal period durations were measured for each wing morph (Fig. 6). In the case of WD morphs of the 06D strain, the mean period of the combined first and second stadia was $5.56 \pm 0.11$ days $(n=27)$. The mean periods of third and fourth stadia were $2.48 \pm 0.12$ days $(n=27)$ and $5.16 \pm 0.14$ days $(n=25)$, respectively, while the total nymphal period was $13.20 \pm 0.16$ days $(n=25)$. In WL males, on the other hand, the first-second, third and fourth stadia were $5.65 \pm 0.08(n=46), 2.37 \pm$ $0.09(\mathrm{n}=46)$, and $3.63 \pm 0.12$ days $(\mathrm{n}=38)$, respectively. The total nymphal period was $11.63 \pm 0.11$ days $(\mathrm{n}=38)$.

In WD males from the HKD strain, the first-second, third and fourth stadia were $5.31 \pm 0.04(\mathrm{n}=128), 2.55 \pm 0.04(\mathrm{n}=128)$, and $4.59 \pm 0.05$ days $(\mathrm{n}=127)$ 
respectively, while the total nymphal period was $12.46 \pm 0.05$ days $(\mathrm{n}=127)$. In IM males, those were $5.22 \pm 0.05(\mathrm{n}=86), 2.47 \pm 0.06(\mathrm{n}=86)$, and $3.93 \pm 0.07$ days $(n=85)$ respectively, while the total period was $11.56 \pm 0.06$ days $(n=85)$.

The first-second stadia did not significantly differ between wing types within each strain (Fig. 6, Tukey-Kramer test, $p>0.05$ ). There were no significant differences at the third stadium between wing types within each strain or between strains (Fig. 6, Tukey-Kramer test, $p>0.05$ ). However, 06D-WD males exhibited the longest the fourth stadium, followed by HKD-WD males; both WL and IM males exhibited the shortest stadium (Tukey-Kramer test, $p<$ 0.05). The fourth stadium of the WL males was about 1.5 days $(29.7 \%)$ shorter than that of the WD males in the 06D strain, while that of IM males was about 0.6 days $(14.4 \%)$ shorter than that of WD males in the HKD strain. Thus, the total nymphal period was affected by the fourth stadium when significant differences between morphs were seen. Among these, 06D-WD males exhibited the longest fourth stadium, followed by HKD-WD males. Both WL and IM males showed the shortest fourth stadium (Tukey-Kramer test, $p<0.05$ ). The total nymphal period of the WL males was about 1.6 days (11.9\%) shorter than that of 06D-WD males, while that of HKD-IM males was about 0.9 days (7.2\%) shorter than that that HKD-WD males. WL males exhibited shorter nymphal periods than IM males, although no significant difference was detected (Tukey-Kramer test, $p>0.05$ ).

\section{Observations of testes}

Based on our observations, testes exhibited no difference among 3 morphs (Fig. S5). All males possessed 6 testes, 2 vas deferens and 2 accessory glands. Although not quantified, testes size also looked similar among 3 morphs. Furthermore, by confocal microscopy observations, no difference in the inner structures was identified. All males, including IM males, produced sperms normally and had normal reproductive ability.

\section{DISCUSSION}

The overall aim of this study is to elucidate the detailed developmental transitions in winged and wingless Acyrthosiphon pisum males observed as a genetic 
polymorphism and to compare them with those seen in the female wing polyphenism. Fortunately, 2 strains producing WD/WL and WD/IM males were available, providing us with an opportunity to examine various male phenotypes.

Based on comparisons of external morphologies, the developmental processes of wing formation and wing bud degradation in the male wing polymorphism are similar to those of the female wing polyphenism (Ishikawa et al. 2008; Fig. S3). In addition, the histological features of wing bud (but not flight muscle) development/degeneration also exhibited some similarities to those of the wing polyphenism (Ishikawa et al. 2008; Fig. 4d, e). A series of microarray analyses (Brisson et al. 2007) suggests that both types of wing polymorphisms share at least some mechanisms that underlie wing bud development.

However, the process of muscle tissue degeneration differed between the female polyphenism and the male polymorphism. It has previously been confirmed that in the female polyphenism, flight muscle primordia degenerate in wingless individuals during early postembryonic development, whereas in winged females muscle degeneration begins after the dispersal flight and continues until the onset of larviposition (Tsuji and Kawada 1987; Ishikawa et al. 2008; Ishikawa and Miura 2009). However, in the case of the male wing polymorphism, no apparent flight muscle degeneration occurs in any wing type (see Tsuji and Kawada 1987, and Fig. 4). Furthermore, no difference in muscle cell differentiation was found between the functional flight muscles in WDs (Fig. 5a) and the non-functional ones in WLs and IMs (Fig. 5c, b), although the degree of flight-muscles development differed among males (Fig. 4, S4). These different processes of muscle degeneration suggest that during the evolution of the female wing polyphenism, flight muscle degeneration appears to have been acquired independently of the male wing polymorphism.

In addition to the flight apparatus, several morphological characteristics (e.g., ocelli, secondary rhinaria, head width and hindtibia length) differ between winged and wingless morphs in the case of the female wing polyphenism (Miyazaki 1987; Ishikawa and Miura 2007). However, no such differences were observed in the case of the male wing polymorphism. Overall, the differentiation between winged and wingless morphs is not nearly as dramatic in the male polymorphism as it is in the female wing polyphenism. 
When comparing the male wing polymorphism with the female wing polyphenism, only the process of wing bud degeneration was similar and many differences were found in other tissues. This suggests that the wing development/degeneration mechanisms were not simply co-opted but partially coopted, probably from the female wing polyphenism to the male wing polymorphism, possibly because different selective pressures might have acted on the 2 types of wing-type alteration mechanisms. Consequently, we must ask what differences regarding selection pressures act on those mechanisms. Although the dispersal ability of wingless females is low, they develop faster, allowing for an earlier onset of larviposition (Tsuji and Kawada 1987; Dixon 1998; Ishikawa and Miura 2009). The difference in time required to reach reproductive maturity will result in differences in total number of offspring (Ishikawa and Miura 2009). While the adaptive significance of winglessness in the female wing polyphenism is mentioned above, the significance of winglessness in males is not clearly understood (Sack and Stern 2007). In this study, there was difference in the nymphal period between winged (WD) and flightless (WL and IM) phenotypes. In particular, the fourth stadium was shortened in the flightless phenotypes, most likely because this period sees the most extensive development of wing buds and flight muscles (Fig. 3, 4). Furthermore, no differences between wing types with respect to fecundity and the developmental extent of testes were identified (Sack and Stern 2007; Fig. S5). Taken together, the differences occurring during the fourth instar are thought to be related to the cost of building flight apparatuses. The adaptive significance of rapid growth in wingless males may provide more opportunities for them to mate with oviparous females, because oviparous females usually aggregate in their habitats to form colonies (Dixon 1998). Thus, the rapid growth conferred by winglessness is thought to be an adaptation.

In this study, we found that nymphal period of intermediate males was shorter than that of winged ones (Fig. 6), suggesting that the trade-off between dispersal ability and developmental period is present even in the 'malformed' individuals. During the process of phenotypic evolution, generally, intermediates are sometimes observed (West-Eberhard 2003), so that the intermediates could represent the transitional phase leading the evolution of wingless phenotype. The time schedules of flight muscle development/degeneration in male wing polymorphism and female wing polyphenism are summarized in Fig. 7. In 
wingless females, wing and flight muscle primordia completely degenerate during early postembryonic development. In winged females, flight-muscle breakdown occurs after the dispersal flight, and energy obtained through the degeneration is re-allocated to reproduction (Tsuji and Kawada 1987; Ishikawa et al. 2008; Ishikawa and Miura 2009; Fig. 7).

As compensation for the loss of flight ability, wingless females can begin reproduction earlier than winged females (Ishikawa and Miura 2009; Fig. 7). No such wing muscle degeneration occurs in the case of male wing polymorphism (Fig. 7). This is probably because the energy required for reproduction is smaller in males; therefore, degeneration of wing muscles and subsequent energy reallocation are not required. However, in male wing polymorphism, the proliferation of flight muscles is suppressed; as a result, the nymphal period is shortened (Fig. 7). Thus, male wing polymorphism and female wing polyphenism possess different regulatory systems for flight-apparatus development/degeneration; this is probably a result of different selection pressures that act on males and parthenogenetic females.

\section{REFERENCES}

Blackman RL (1987) Reproduction, cytogenetics and development. In: Minks AK and Harrewijin P (eds) Aphids, Their Biology, Natural Enemies and Control. Elservier, Amsterdam, pp 163-195

Braendle C, Caillaud MC, Stern DL (2005a) Genetic mapping of aphicarus - a sex-linked locus controlling a wing polymorphism in the pea aphid (Acyrthosipon pisum). Heredity 94:435-442

Braendle C, Davis GK, Brisson JA, Stern DL (2006) Wing dimorphism in aphids. Heredity 97:192-199

Braendle C, Friebe I, Caillaud MC, Stern DL (2005b) Genetic variation for an aphid wing polyphenism is genetically linked to a naturally occurring wing polymorphism. Proc R Soc B 272:657-664

Brisson JA, Davis GK, Stern DL (2007) Common genome-wide patterns of transcript accumulation underlying the wing polyphenism and polymorphism in the pea ahid (Acyrthosiphon pisum). Evol Dev 9:338-346 
Brisson JA (2010) Aphid wing dimorphisms: linking environmental and genetic control of trait variation. Phil Trans R Soc B 365:605-616

Caillaud MC, Boutin M, Braendle C, Simon JC (2002) A sex-linked locus controls wing polymorphism in males of the pea aphid, Acyrthosiphon pisum (Harris). Heredity 89:346-352

Dixon AFG (1998) Aphid Ecology. Chapman \& Hall, London.

Dudley R (2002) The Biomechanics of Insect Flight: Form, Function, Evolution. Princeton University Press, Princeton.

Harrison RG (1980) Dispersal polymorphisms in insects. Annu Rev Ecol Syst 11:95-118

Ishikawa A, Miura T (2007) Morphological difference between wing morphs of two Macrosiphini aphid species, Acyrthosipon pisum and Megoura crassicauda (Hemiptera, Aphididae). Sociobiology 50:881-893

Ishikawa A, Hongo S, Miura T (2008) Morphological and histological examination of polyphenic wing formation in the pea aphid Acyrthosiphon pisum (Heteroptera, Hexapoda). Zoomorphology $127: 121-133$

Ishikawa A, Miura T (2009) Differential regulations of wing and ovarian development and heterochronic changes of embryogenesis between morphs in wing polyphenism of vetch aphid. Evol Dev 11:680-688

Ishikawa A, Ogawa K, Gotoh H, Walsh TK, Tagu D, Brisson JA, Rispe C, Jaubert-Possamai S, Kanbe T, Tsubota T, Shiotsuki T, Miura T (2012) Juvenile hormone titre and related gene expression during the change of reproductive modes in the pea aphid. Insect Mol Biol. 21:49-60

Kring JB (1977) Structure of the eyes of the pea aphid, Acyrthosiphon pisum. Ann Entomol Soc Am 70:855-860

Lamb RJ, Pointing PJ (1972) Sexual morph determination in the aphid, Acyrthosiphon pisum. J Insect Physiol. 18:2029-2042

Lees AD (1966) The control of polymorphism in aphids. Adv Insect Physiol 3:207-277

Marcovitch S (1923) Plant lice and light exposure. Science 58:537-538

Miyazaki M (1987) Forms and Morphs of aphids. In: Minks AK and Harrewijin P (eds). Aphids, Their Biology, Natural Enemies, and Control. Elservier, Amsterdam pp 27-50 
Orlando E (1974) Sex determination in Megoura viciae Buckton (Homoptera Aphididae). Monit. Zool. Ital. 8:61-70

Roff DA (1990) The evolution of flightlessness in insects. Ecol. Monogr. 60:389-421

Roff DA, Fairbairn DJ (1991) Wing Dimorphisms and the evolution of migratory polymorphisms among the Insecta. Am Zool 31:243-251

Sack C, Stern DL (2007) Sex and Death in the male pea aphid, Acyrtosiphon pisum: The lifehistory effects of a wing dimorphism. J Insect Sci 7:1-9

Smith MAH, MacKay PA (1989) Genetic variation in male alary dimorphism in populations of pea aphid, Acyrthosiphon pisum. Entomol Exp Appl 51:125-132

Tsuji H, Kawada K (1987) Development and degeneration of wing buds and indirect flight muscle in the pea aphid (Acyrthosiphon pisum (Harris)). Jpn J Appl Ent Zool 31:247-252

Wagner DL, Liebherr JK (1992) Flightlessness in insects. Trends Ecol. Evol. 7:216-220

Wilkinson TL, Ishikawa H (2000) Injection of essential amino acids substitutes for bacterial supply in aposymbiotic pea aphids (Acyrthosiphon pisum). Entomol. Exp. Appl. 94:85-91

Zera AJ, Denno RF (1997) Physiology and ecology of dispersal polymorphism in insects. Annu Rev Entomol 42:207-230.

\section{FIGURE LEGENDS}

Fig. 1. Schematic diagram of the annual life cycle of A. pisum (a) and the genetic mechanism for wing-type determination in the male wing polymorphism (b). a A. pisum reproduces by thelytokous parthenogenesis under spring and summer day lengths and temperatures. Males and sexual females (oviparous females) appear only in the fall and produce fertilized eggs for overwintering. Viviparous females express the wing polyphenism, and males express the genetic wing polymorphism. b Male wing types are determined by a locus called api on the $\mathrm{X}$ chromosome. In the case of heterozygosity, the wing type of the progeny is determined by which $\mathrm{X}$-chromosome is discarded. $F$ fundatrix, $W V$ winged viviparous female, $W L V$ wingless viviparous female, $S$ sexupara, $O$ oviparous female, $W M$ winged male, WLM wingless male, IMM wingedwingless intermediate male, $D E$ diapausing eggs. 
Fig. 2. Comparisons of overall morphology and colors among wing types in the male wing polymorphism; a winged (WD); b-d intermediate (IM); e wingless (WL). The notum colors of winged and wingless males are dark brown (a) and green (similar to abdominal parts; e) of their surface respectively; the notum color of intermediate males varies from yellow-green (d) to yellow-brown (b, c). All male morphs possess fully developed ocelli. Arrowheads indicate deformed wings, and arrows show nota.

Fig. 3. Detailed morphological comparisons among wing types in the male wing polymorphism throughout postembryonic development observed by scanning electron microscope (SEM). a first instar; b second instar; c-e third instar; $\mathbf{f}-\mathbf{i}$ fourth instar; $\mathbf{j}-\mathbf{m}$ fifth instar. $\mathbf{c}, \mathbf{f}, \mathbf{j}$ winged line $(W D)$; $\mathbf{d}, \mathbf{g}, \mathbf{h}, \mathbf{k}, \mathbf{l}$ intermediate line $(I M) ; \mathbf{e}, \mathbf{i}, \mathbf{m}$ wingless line $(W L)$. The 3 wing types can be identified by their strain and thoracic morphology (wing buds) from the third instar. Arrowheads indicate wing buds (or pads), and arrows show membranous wings.

Fig. 4. Histological comparisons of the postembryonic development of flight apparatus among wing types in the male wing polymorphism and the female wing polyphenism. a-c male wing polymorphism (a: winged line, b: intermediate line, and c: wingless line). d, e female wing polyphenism (d: winged line, e: wingless line). f transverse planes of thoracic segment (left: $W D$, right: $I M$ or $W L)$. g sagittal planes of thoracic segment (after Tsuji and Kawada 1987). All male morphs possess flight muscles, while only winged females do. Panels on the right of sections are 3D reconstructions of flight muscles. Sections were stained with hematoxylin and eosin. $3 r d$ third instar, 4th fourth instar, 5th fifth instar, $D V M$ dorsoventral muscle, $D L M$ dorsal longitudinal muscle, $O D M$ oblique dorsal muscle.

Fig. 5. Flight muscle (DVM) development in the male wing polymorphism. a winged line; b intermediate line; c wingless line. Flight muscle primordia with a large number of myoblasts are seen in the third instar, and differentiated muscle fibers are seen in the fourth instar. Muscles are completely developed and numerous fibers are clearly seen in the fifth instar. There are no clear differences regarding muscle cell differentiation between functional flight muscles in WDs (a) and non-functional ones in WLs (c) and IMs (b). Sections were stained with hematoxylin and eosin. $3 r d$ third instar, 4 th fourth instar, 5th fifth instar.

Fig. 6. Comparisons of the nymphal periods among wing types in the male wing polymorphism. The different letters $(\mathrm{a}-\mathrm{c})$ denote significant differences between groups (Tukey-Kramer test, $p<0.05)$. Apparent differences in nymphal periods were detected in the fourth instar. WD winged line, $W L$ wingless line, $I M$ intermediate line. 
Fig. 7. Schematic diagram of flight muscle development in A. pisum. In winged viviparous adult females, flight muscle breakdown and energy reallocation occur after the migration flight; these events occur during the nymphal period in wingless viviparous females. On the other hand, in males the degeneration of flight muscles and muscle primordia do not occur. The growth of winged males with fully grown flight muscles requires a longer period than that for wingless males with poorly developed flight muscles. 


\section{a}

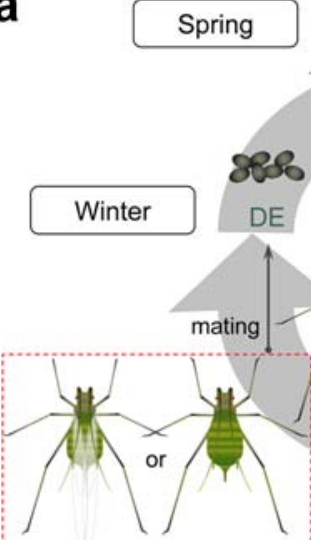

WM

WLM

Wing polymorphism

(Genetic) b
Summer

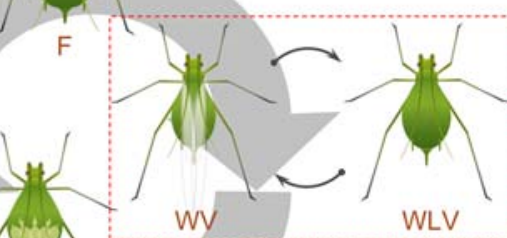

Wing polyphenism

(Environmental)
Fall
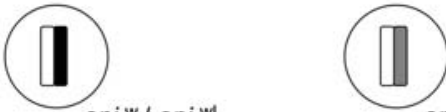

api ${ }^{\mathrm{w}} / \mathrm{api} \mathrm{w}^{\mathrm{w}}$

opi $/$ api im $\bigwedge$

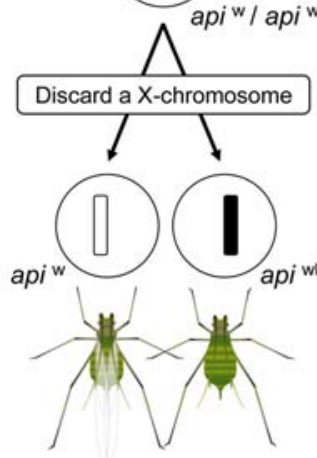

WM

06D strain

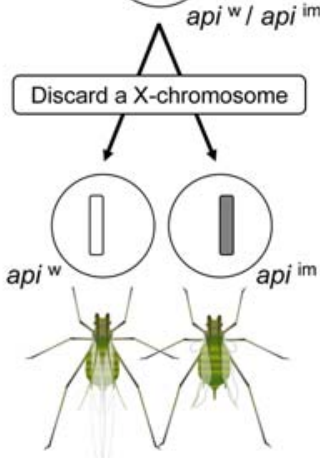

WM

IMM
HKD strain 
Male

a) winged

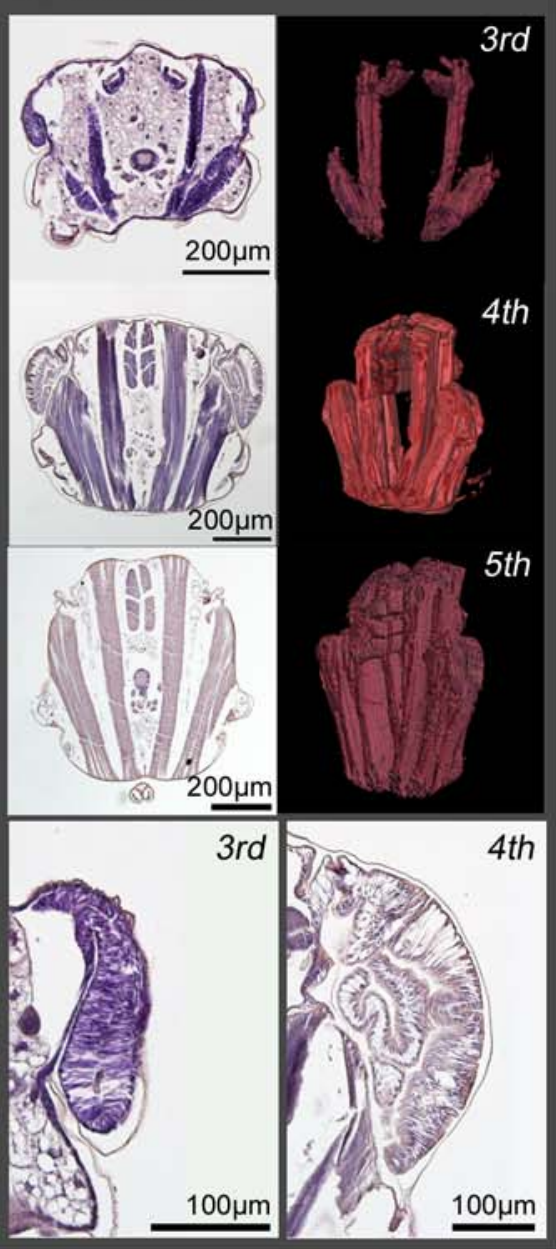

b) intermediate
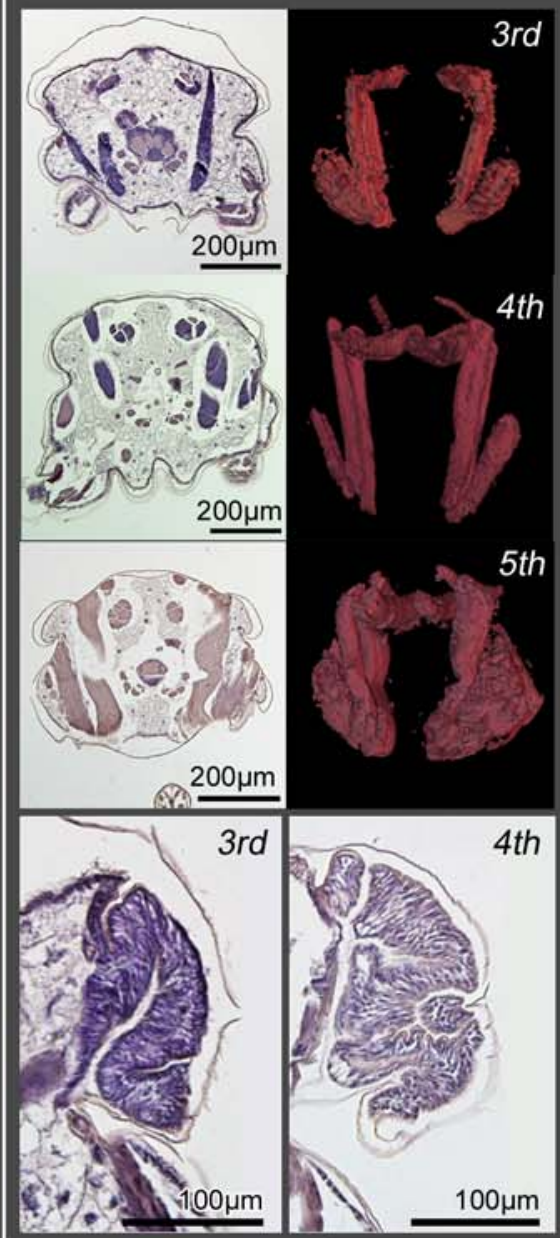

4th c) wingless
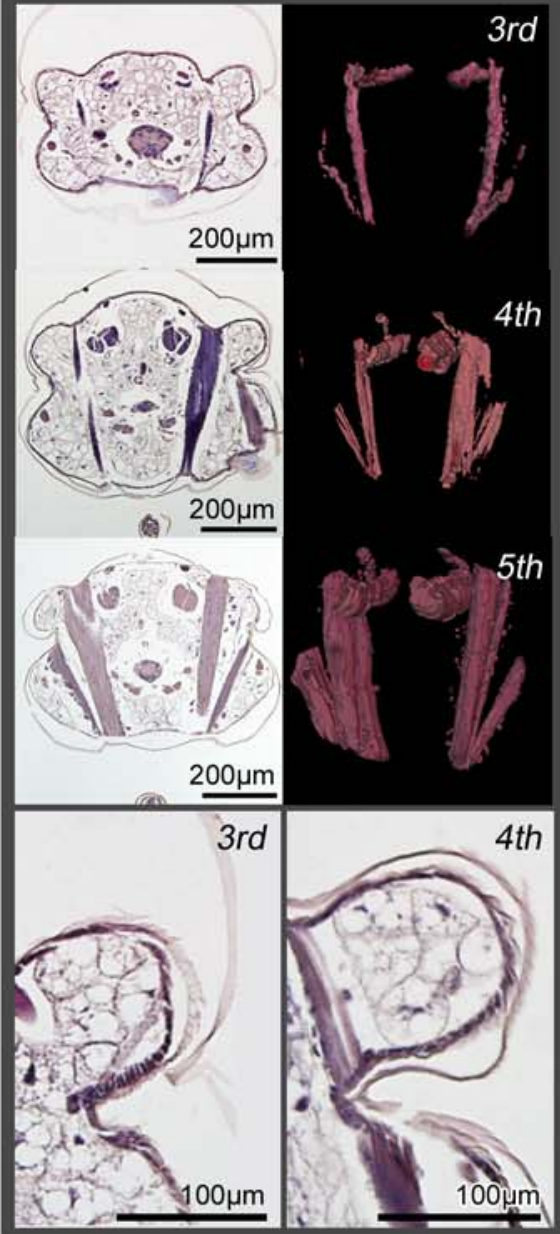

d) winged

E)

\section{$200 \mu \mathrm{m}$}

Viviparous female

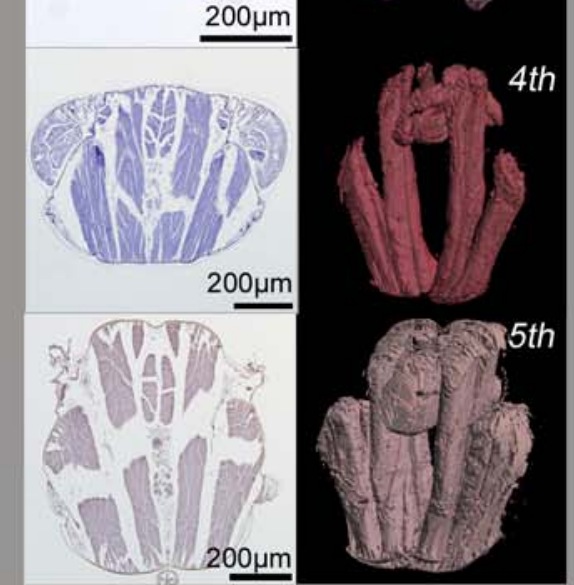

e) wingless

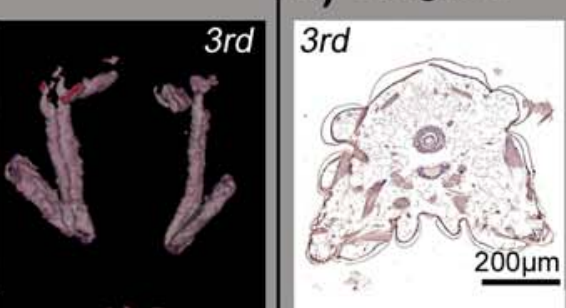

4th

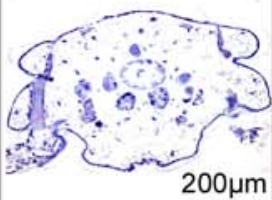

5th

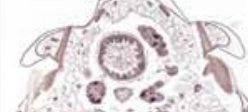

f

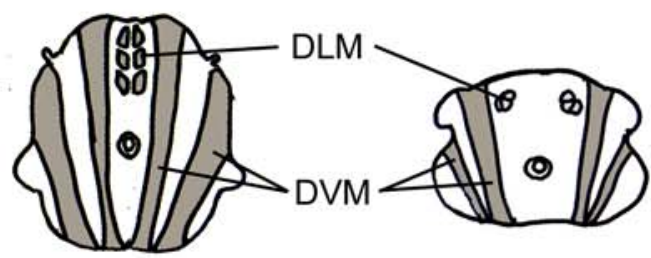

g
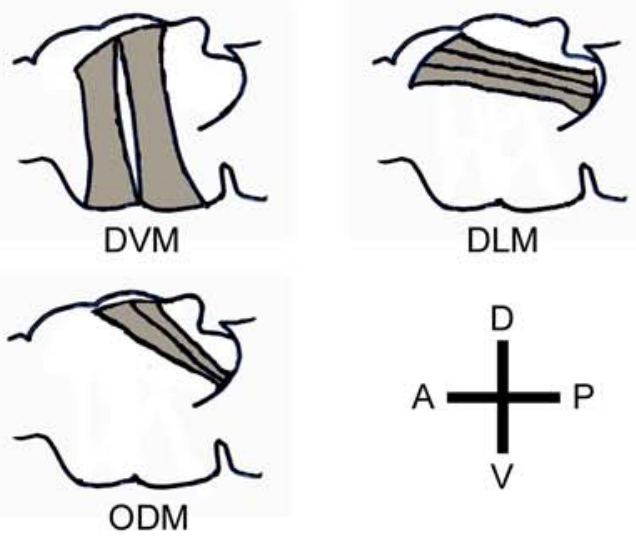


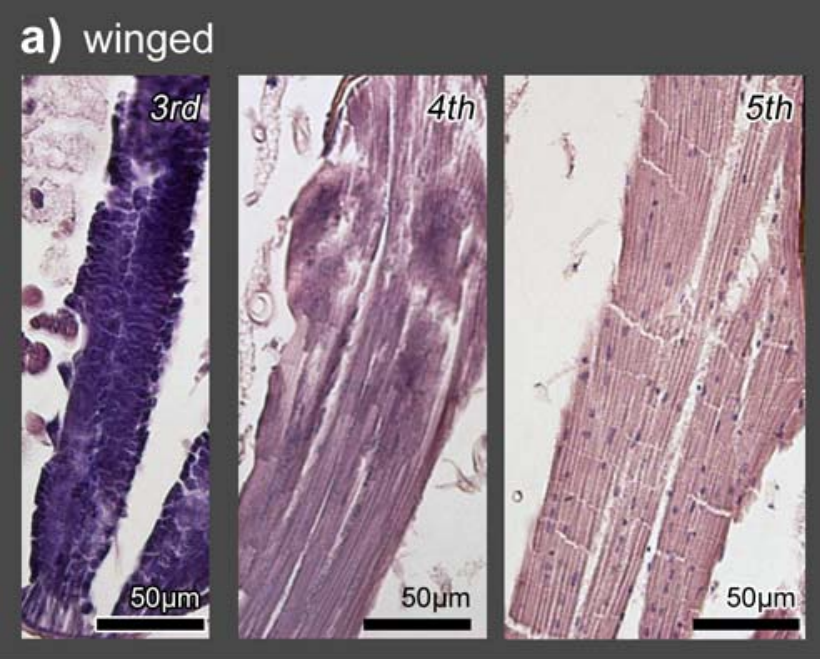

\section{b) inetermediate}
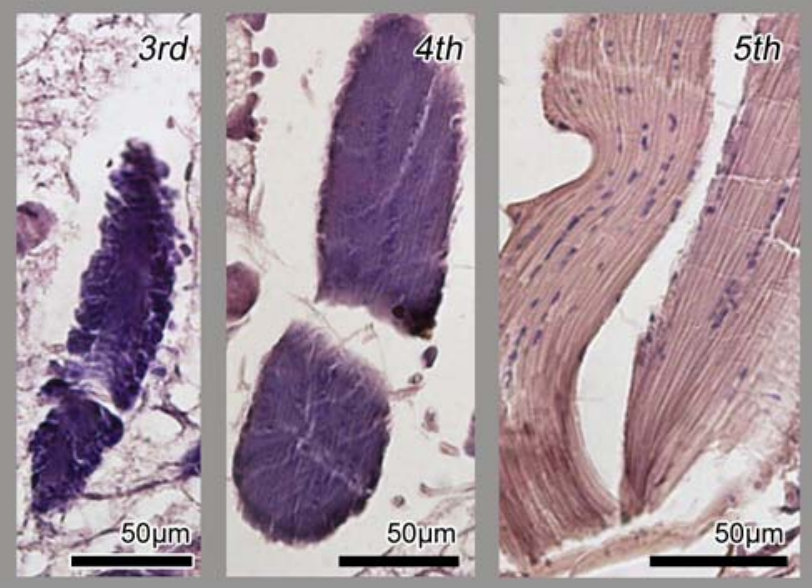

\section{c) wingless}
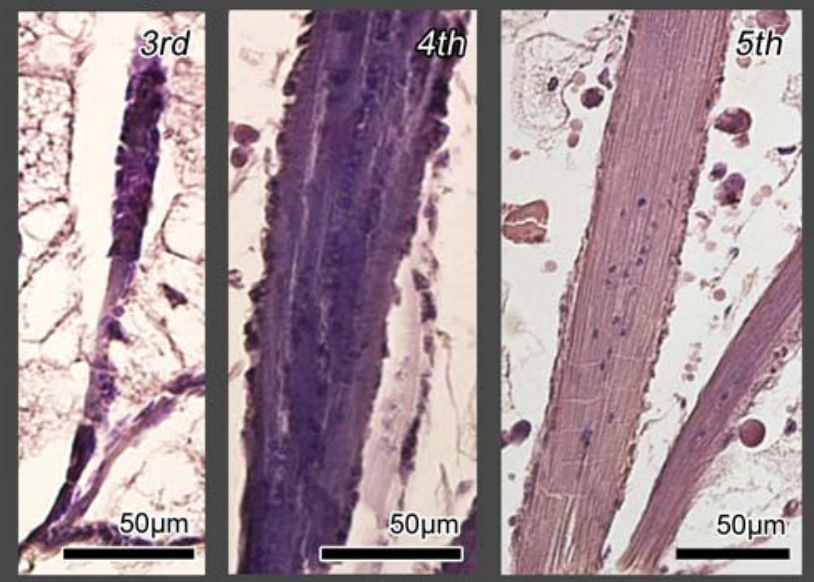
1st - 2nd instar $\quad 3$ rd instar $\quad$ 4th instar

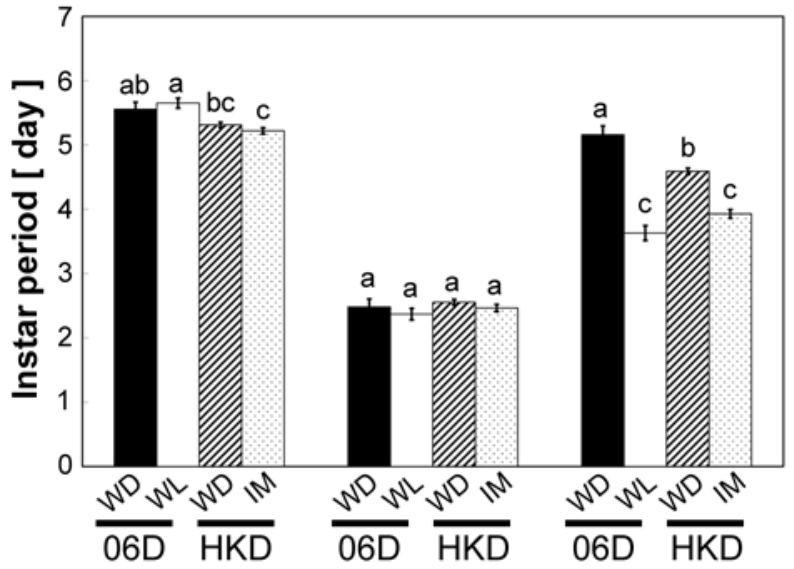




\section{Genetic polymorphism (Male)}

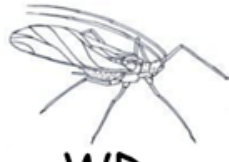

WD

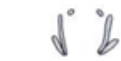

Muscle primordia

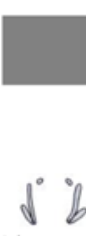

Muscle

primordia Growth (less)

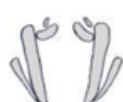

Growth

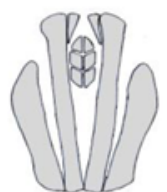

Differentiation

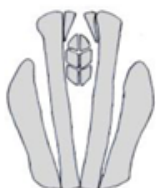

No degeneration

\section{Nymphal period}

Flight for mating

\section{Polyphenism (Viviparous female)}

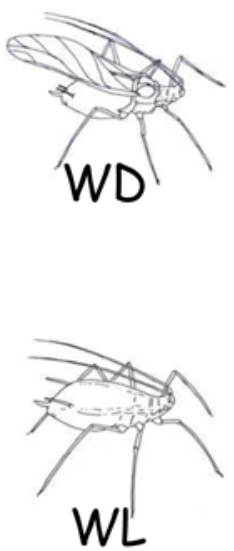

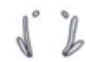

Muscle primordia

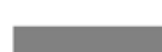

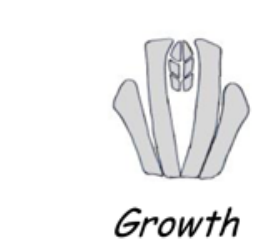

\section{Nymphal period}

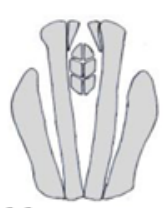

Differentiation

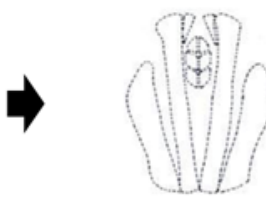

Degeneration

No degeneration

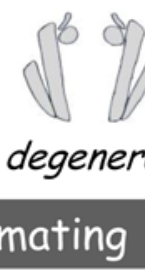

Walk for mating

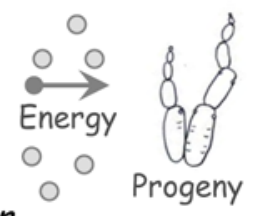

\section{Flight}

Larvipositon

\section{Iis}

Muscle Degeneration primordia

\section{Nymphal period}

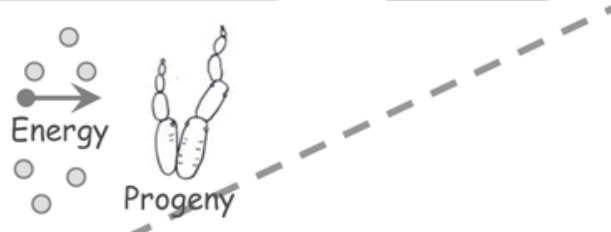

Larviposition 
Table 1 Comparisons of the head width, mesonotum width, mesonotum length and right hindtibia length among three morphs in the male A. pisum.

\begin{tabular}{|c|c|c|c|c|}
\hline Strain & Morph & Head width & Mesonotum width ${ }^{\#}$ & Mesonotum length ${ }^{\#}$ Hindtibia length \\
\hline \multirow[t]{2}{*}{ 06D } & WD & $0.525 \pm 0.003(25)$ & $0.698 \pm 0.008(25)^{\mathrm{A}}$ & $0.649 \pm 0.008(25)^{\text {a }} 2.23 \pm 0.04(22)$ \\
\hline & WL & $0.520 \pm$ & 0.59 & 5) ${ }^{c} 2.2$ \\
\hline \multirow[t]{2}{*}{ HKD } & WD & & 0.686 & $0.648 \pm 0.010(17)^{\text {a }} 2.22 \pm 0$. \\
\hline & $v_{1}$ & $0.517 \pm 0.003(46)$ & $0.662 \pm 0.009(46)^{\mathrm{B}}$ & $0.420 \pm 0.012(46)^{b} 2.24 \pm 0.02(43)$ \\
\hline
\end{tabular}
Values are means [mm] \pm SE $(\mathrm{n})$. \# The different letters $(A-C$ and $a-c)$ denote significant differences between each group (Tukey-Kramer test, $p<0.05$ ). 